\author{
G U E S T E D I T O R I A L
}

\title{
Special Section on Nonlinear Image Processing
}

\author{
Jaakko T. Astola \\ Tampere University of Technology \\ Signal Processing Laboratory \\ P.O. Box 553 \\ SF-33101 Tampere, Finland \\ E-mail: jta@cs.tut.fi

\section{Junior Barrera} \\ University de São Paulo \\ Instituto de Mathematica e Estatistica \\ CP 20570, Armando de Salles Oliveira \\ Rua do Matao 1010 \\ São Paulo 01452-990, Brazil
}

Complex image processing tasks such as coding, restoration, or segmentation of digital images cannot be efficiently performed using linear signal processing methods only. The success of many nonlinear image processing methods in a variety of important practical applications and several fundamental advances in the theory of nonlinear image and signal processing have made nonlinear image processing one of the most active fields in electronic imaging and signal processing.

In the last decade, these research efforts have been quite successful. Not only have new techniques emerged, but also deep connections have been found between the various nonlinear image processing methods that have grown from different origins. Our understanding of these methods has evolved to the level where rigorous design procedures based on solid mathematical modeling of the target application have become possible. The recent advances in probabilistic optimization and the ever increasing computing power have made learning methods one of the best tools for practical nonlinear filter design. This special section reflects these general tendencies.

An exceptionally fruitful framework in nonlinear image processing is mathematical morphology. It is a theory that studies the decomposition of filters in terms of more elementary filters, which is particularly useful for the description of shape transformations. Since its in- troduction in the 1960 s by Matheron and Serra, mathematical morphology has proved its power by solving hundreds of image processing problems.

In the paper "Morphological partitioning of multispectral images," Soille gives a new application of mathematical morphology for the segmentation of colored or general multispectral images. A classical technique for the morphological segmentation of images is the filtering of the morphological gradient of the image and the computation of its watershed. A critical point of this procedure is the filtering of the gradient, which depends on markers for the relevant objects of the image. Hence, methods for the determination of markers become crucial for the performance of the procedure. In the context of multispectral images, a new technique for the computation of markers based on morphological transformations of the histogram of the image is proposed. In the paper "Adaptive reconstructive $\tau$-openings: convergence and the steady-state distribution," Chen and Dougherty address the fundamental problems of statistical modeling of images and morphological methods. Also under the framework of mathematical morphology, in the paper "Morphological scale-space preserving transforms in many dimensions," Bangham et al. propose a new morphological filter that has many useful properties such as preservation of scale-space causality. Filters with this property are quite im- portant, for example, in computer systems that perform pattern recognition such as optical character recognition.

A fundamental problem in image processing is the automatic design of filters. This problem is usually formulated in the context of statistical optimization. The search space is a family of filters represented in a given decomposition structure, and the goal is to find the best filter of the family according to some statistical measure of the behavior of the filters. Once filters are presented on fixed decomposition structures, the optimization procedures consist of finding the right parameters in the decompositions. In many applications, families of morphological filters are chosen as the search space over which optimization is performed. In the paper "Training-based optimization of soft morphological filters," Koivisto et al. present techniques of optimal statistical designing over the family of soft morphological filters. They apply modern combinatorial optimization, simulated annealing and genetic algorithms for the search of optimal filters that perform particular image processing tasks.

In the paper "Efficient nonlinear transform methods for image processing," Atourian et al. study filters created by their decomposition in terms of linear combinations of stack filters. This type of filter includes many useful families of filters such as the threshold Boolean filters and the L-filters. They also present an efficient algorithm for 
finding optimal filter coefficients under the mean square error criterion.

It would be ideal to optimize both the filter and the decomposition used to represent the optimal filter; that is, after finding the optimal filter, its decomposition structure is changed until a structure that is well adapted for implementation in the available architecture is reached. In the paper "Set operations on closed intervals and their applications to the automatic programming of morphological machines," Barrera and Salas partially address this problem under the framework of mathematical morphology. They propose a method that transforms any representation of a morphological filter into its canonical form (i.e., a supremum of hit-miss transformations). This method is applied for the optimization of the representation of some filters and for an automatic proof of the equivalence between representations of filters.

Nonlinear energy measures have received much attention in recent years, especially in speech processing where so-called Tieger energy can be used efficiently to extract many useful properties of the speech signal. In the paper "Nonlinear unsharp masking methods for image contrast enhancement," Ramponi et al. show that Tieger energy is also very useful in the processing of images.
Multiresolution analysis is a very active field of signal and image processing as reflected by the vast recent literature, e.g., on wavelets, multirate signal processing, and subband coding techniques. Similar results appear in nonlinear image processing as the morphological opening and closing. In the paper "Nonlinear multiresolution decomposition with applications in image restoration," Anandakumar and Kassam present a nonlinear multiresolution decomposition scheme based on order statistic filters. They apply their decomposition for the restoration of images and use nonlinear techniques for attenuating the components created.

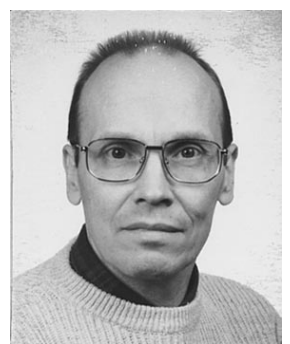

Jaakko T. Astola received the $\mathrm{BSc}$, MSc, Licentiate, and $\mathrm{PhD}$ degrees in mathematics from Turku University, Finland, in 1972, 1973, 1975 and 1978 , respectively. From 1976 to 1977 he was a research assistant at the Research Institute for Mathematical Sciences of Kyoto University, Kyoto, Japan. Between 1979 and 1987 he was with the Department of Information Technology, Lappeenranta University of Technology, Lappeenranta, Finland, holding various teaching positions in mathematics, applied mathematics, and computer science. From 1988 to 1993 he was an associate professor in applied mathematics at Tampere University, Tampere, Finland. Currently he is professor of digital signal processing at Tampere University of Technology. His research interests include image and signal processing, coding theory, and statistics.

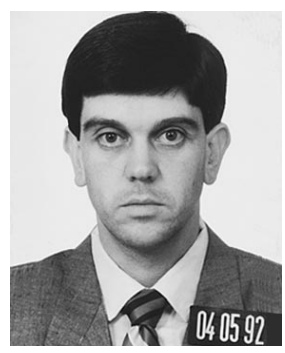

Junior Barrera received the degree of doctor in automatic control and systems from the University of São Paulo in 1992. Since June 1992, he has been an assistant professor at the Department of Computer Science of the Institute of Mathematics and Statistics of the University of São Paulo. In the last 10 years he has worked in the field of image analysis by mathematical morphology. He has several international publications with theoretical and applied contributions. He is coauthor of the proof that any lattice operator can be decomposed in terms of elementary operators of mathematical morphology. He is also coauthor of the MMach toolbox, a very popular software for image analysis by mathematical morphology. 